\title{
Intra-arterial calcium stimulation test with hepatic venous sampling for preoperative diagnosis of a large insulinoma in an obese young man
}

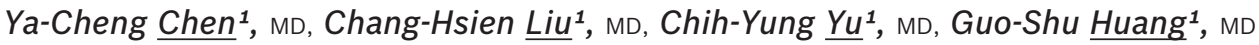

\begin{abstract}
Herein, we report the case of a large benign insulinoma in an obese young man with a three-year history of asymptomatic hypoglycaemia. He presented to our outpatient department with a two-week history of dizziness and morning cold sweats. A random serum glucose test revealed hypoglycaemia. Upon admission, computed tomography and magnetic resonance imaging of the abdomen with intravenous contrast media showed an enhancing mass lesion in the uncinate process of the pancreas. To confirm the diagnosis, an intra-arterial calcium stimulation test with hepatic venous sampling was performed for preoperative localisation and to exclude the presence of occult insulinomas. The patient underwent an exploratory laparotomy, with successful resection of the pancreatic head tumour. Histology confirmed the diagnosis of insulinoma. The patient's postoperative recovery was uneventful, and he has not developed further episodes of hypoglycaemia three years post surgery.
\end{abstract}

Keywords: computed tomography, insulinoma, intra-arterial calcium, magnetic resonance, stimulation test with hepatic venous sampling

\section{INTRODUCTION}

An insulinoma is a rare neuroendocrine tumour of the pancreas, with a reported incidence of about $1-4$ per million per year, ${ }^{(1,2)}$ a female predominance $(60 \%)^{(3)}$ and a mean age at diagnosis of about 50 years. ${ }^{(3)}$ Insulinomas make up $70 \%-80 \%$ of all functional neuroendocrine tumours of the pancreas. Most insulinomas are benign, solitary and small; at diagnosis, $90 \%$ of insulinomas measure less than $2 \mathrm{~cm}$ and only $4 \%$ are larger than $3 \mathrm{~cm} \cdot{ }^{(4,5)}$ Malignant involvement increases with tumour size, and about one-third metastasise to the liver. ${ }^{(3)}$ Insulinomas can cause considerable morbidity and are diagnosed when increases in insulin and C-peptide levels in association with hypoglycaemia are observed. In obese adults, the leptin level is elevated, leading to a decrease in plasma insulin level. Thus, in obese adults with asymptomatic hypoglycaemia, the diagnosis of insulinoma is often delayed.

Herein, we report the case of an obese young man with a 6-cm pancreatic tumour detected by computed tomography (CT) and magnetic resonance (MR) imaging. Insulinoma was diagnosed using an intra-arterial calcium-stimulating test with hepatic venous sampling (IACS), which showed no occult lesions.

\section{CASE REPORT}

A 30-year-old man with asymptomatic hypoglycaemia, diagnosed during a routine health examination about three years previously, presented to our outpatient department with a two-week history of intermittent dizziness and morning cold sweats. A random serum glucose test revealed hypoglycaemia (blood glucose level $57 \mathrm{mg} / \mathrm{dL}$ ) and body weight gain of about 5-6 kg in the preceding six months. At admission, the patient measured $183 \mathrm{~cm}$ in height and weighed $94 \mathrm{~kg}$, giving a body mass index of $33.6 \mathrm{~kg} / \mathrm{m}^{2}$. He showed normal vital signs with no anomalous findings in physical and neurological examinations. Biochemical measurements, including complete blood count and levels of thyroid hormone, parathyroid hormone, calcium and prolactin, were also normal. In a seven-hour fasting test, the patient developed hypoglycaemic symptoms such as dizziness and cold sweats, low plasma glucose level $(32 \mathrm{mg} / \mathrm{dL})$, and abnormally elevated levels of insulin $(8.1 \mu \mathrm{lU} / \mathrm{mL})$ and C-peptide $(1.8 \mathrm{ng} / \mathrm{mL})$.

Dynamic CT of the abdomen revealed a mild lobulated homogeneous mass measuring $6.0 \mathrm{~cm} \times 4.7 \mathrm{~cm}$ in size in the uncinate process of the pancreas, with strong enhancement in the arterial phase, persistent enhancement in the portal venous phase, and attenuation similar to normal pancreas in the delayed phase (Fig. 1). MR imaging showed the mass to be mildly hypointense on T1-weighted (T1-W) images and mildly hyperintense on T2-weighted (T2-W) and diffusion-weighted (DW) images (Fig. 2). As in the CT images, MR images showed homogeneous enhancement on dynamic study, and hence, a neuroendocrine tumour was suspected.

To localise the tumour, an IACS was performed on the patient. A 4.1 French (Fr) catheter with a side hole at the tip was positioned through the left femoral vein into the middle hepatic vein. Catheterisation with another 4.1 Fr catheter was done for the right femoral artery, after which standard pancreatic arteriography was performed with selective injections of nonionic contrast agent into the superior mesenteric artery (SMA), gastroduodenal artery (GDA), proper hepatic artery (PHA), proximal splenic artery (PSA) and distal splenic artery (DSA). Following each selective arteriogram, $10 \%$ calcium gluconate solution was diluted with normal saline into a $5 \mathrm{~mL}$ bolus and injected into the selective artery at a dose of $0.010-0.025 \mathrm{mEq} \mathrm{Ca}{ }^{2+} / \mathrm{kg}$. A twofold or higher

${ }^{1}$ Department of Radiology, Tri-Service General Hospital, National Defense Medical Centre, Taipei, Republic of China (Taiwan)

Correspondence: Dr Chang-Hsien Liu, Assistant Professor, Department of Radiology, Tri-Service General Hospital, National Defense Medical Centre, No. 325, Sec 2, ChengKung Road, Neihu 114, Taipei, Republic of China (Taiwan). cute610627@yahoo.com.tw 

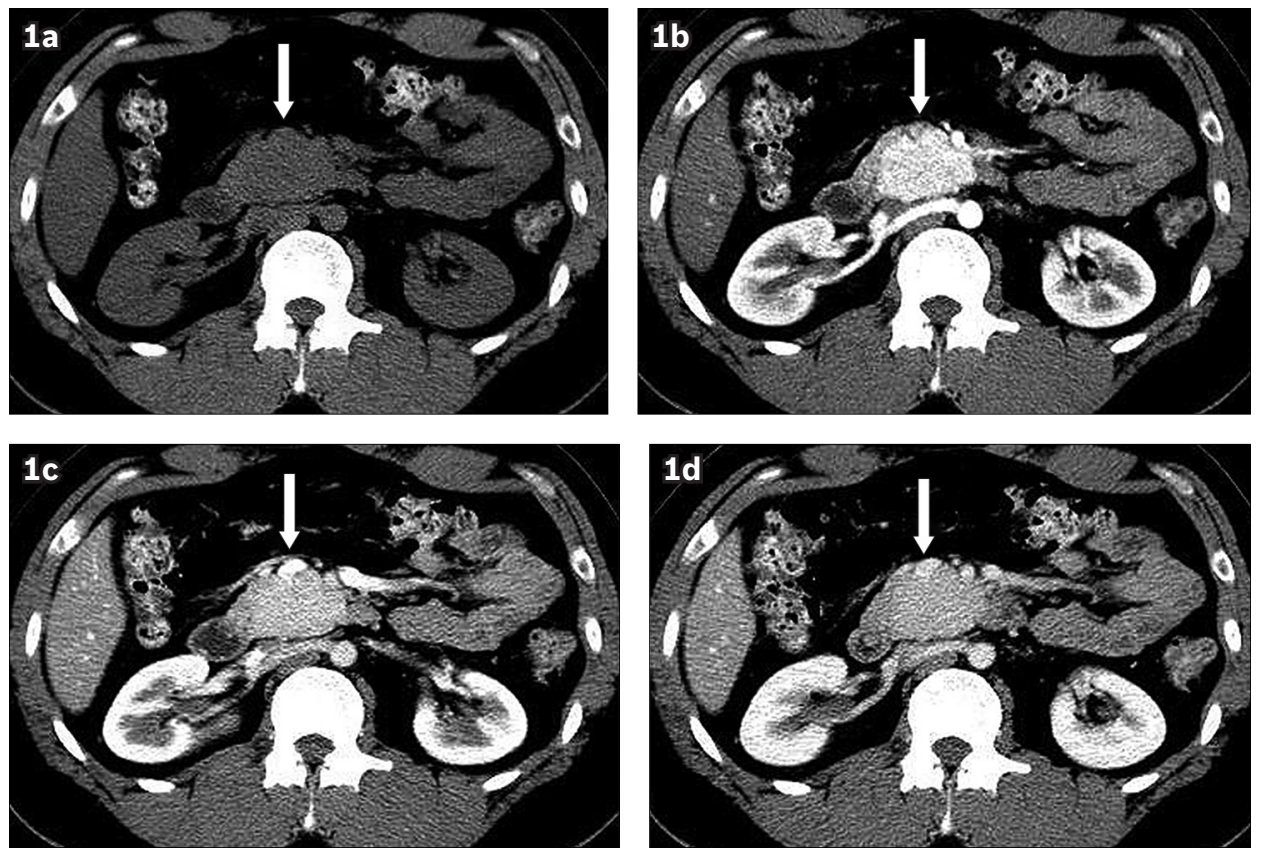

Fig. 1 Non-contrast-enhanced axial CT images show (a) a 6-cm mass in the uncinate process of the pancreas (arrow); (b) strong enhancement in the arterial phase (arrow); (c) persistent enhancement in the portal venous phase (arrow); and (d) contrast washout of the mass, with attenuation similar to the normal pancreas in the delayed phase (arrow).
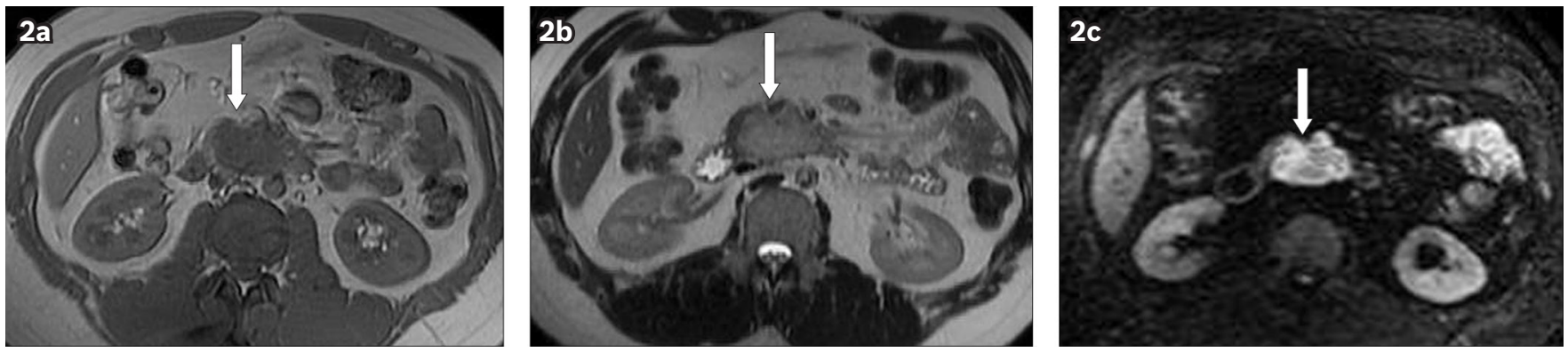

Fig. 2 MR imaging of the abdomen. (a) Axial T1-W MR image shows mild hypointensity of the lesion over the uncinate process of the pancreas (arrow); and axial (b) T2-W fat-saturated and (c) diffusion-weighted MR images show mild hyperintensity of the lesion (arrow).
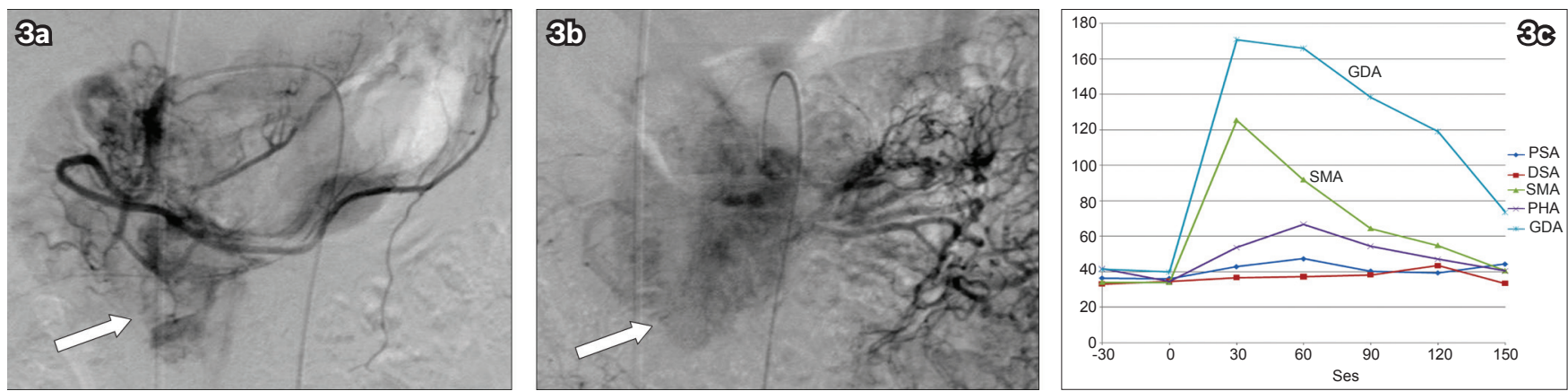

Fig. 3 (a) Gastroduodenal and (b) superior mesenteric angiography images show a hypervascular lesion in the uncinate process of the pancreas (arrow); and (c) graph of intra-arterial calcium stimulating test with hepatic venous sampling results shows a twofold or higher increase of the insulin level from baseline in the SMA and GDA. DSA: distal splenic artery; GDA: gastroduodenal artery; PHA: proper hepatic artery, PSA: proximal splenic artery; SMA: superior mesenteric artery

step-up in hepatic vein insulin concentration from the baseline at 30 s, 60 s, 90 s, 120 s, or 180 s after the arterial calcium injection was defined as a positive response. ${ }^{(2,4,6-11)}$

During the IACS, pancreatic angiography revealed a hypervascular mass lesion in the uncinate process of the pancreas, which the venous sampling test showed as positive during calcium injection into the SMA and GDA (Fig. 3). A diagnosis of insulinoma was reached. No occult lesion over the pancreas or metastatic lesion in the liver was identified.

Following the diagnosis of insulinoma, localisation of the lesion and confirmation of the absence of other occult lesions, a pylorus-preserving pancreaticoduodenectomy (Whipple procedure) was performed. The resected tumour was well encapsulated, without extracapsular invasion in the pancreatic 
uncinate process. Immunohistochemical study showed that the tumour was positive for insulin (Fig. 4). This finding was consistent with insulinoma. Postoperatively, the patient's plasma glucose level and insulin concentrations returned to normal, and his symptoms resolved without further hypoglycaemic events in the subsequent three years.

\section{DISCUSSION}

Insulinoma, a rare endocrine tumour, is the most common cause of hyperinsulinaemic hypoglycaemia in adults. It usually presents between the ages of 25 and 75 years and shows a female predominance. Insulinoma is diagnosed on the basis of Whipple's triad, which includes a low plasma glucose level, the appearance of symptoms during fasting, and symptom relief after glucose administration. ${ }^{(3,12)}$ Clinically, insulinoma presents with oversecretion of insulin, neuropsychiatric symptoms (e.g. loss of consciousness, confusion and dizziness), cardiovascular symptoms (e.g. sweating, palpitation and tachycardia) and obesity. ${ }^{(12)}$

Insulinomas are relatively small tumours with a mean size of $1.5 \mathrm{~cm}-90 \%$ of insulinomas are less than $2 \mathrm{~cm}$ at the time of diagnosis. ${ }^{(1)}$ Most insulinomas are benign, but tumours larger than $3 \mathrm{~cm}$ are associated with malignancy. ${ }^{(5,13)}$ Insulinomas can be classified as sporadic or familial. Sporadic insulinomas are usually solitary, whereas familial insulinomas occur as multiple lesions and are associated with multiple endocrine neoplasia type 1 (MEN-1) syndrome. The peak incidence of sporadic insulinomas occurs between the third and fifth decades of life and women are more frequently affected. In the case of familial MEN-1, the peak incidence is reported to occur during the third decade, with a male predominance. In the current study, the patient was a young man with a $6-\mathrm{cm}$ sporadic pancreatic insulinoma, which is an uncommon clinical presentation. Currently, there are no histological or morphological criteria that distinguish benign insulinomas from malignant ones. The diagnosis for malignancy can only be made by detecting the presence of metastases in the liver or regional lymph nodes, or evidence of local invasion. ${ }^{(13)}$

A number of techniques are available to detect pancreatic insulinomas, including abdominal ultrasonography (US), CT, MR imaging, nuclear medicine, endoscopic ultrasonography (EUS), percutaneous port venous sampling and IACS..$^{(3,10,14)}$ Abdominal US, one of the first imaging techniques to be used to detect insulinomas, has a poor detection rate due to the small size of the tumour, interference by other intra-abdominal organs, and obesity. CT is a safe, noninvasive and operator-independent imaging modality that can detect the location of a tumour, its relationship to vital structures and the presence of metastases. Neuroendocrine tumours such as insulinomas, glucagonomas or VIPomas are hypervascular lesions and thus show good enhancement on arterial phase images. Like CT, MR imaging is safe and noninvasive, with the ability to detect the number and location of tumours, as well as the presence of metastases. MR imaging is, however, more sensitive than $\mathrm{CT}$ in detecting lesions. On MR imaging, neuroendocrine tumours can be visualised as low signal intensity on $\mathrm{T} 1-\mathrm{W}$ images, high signal intensity on

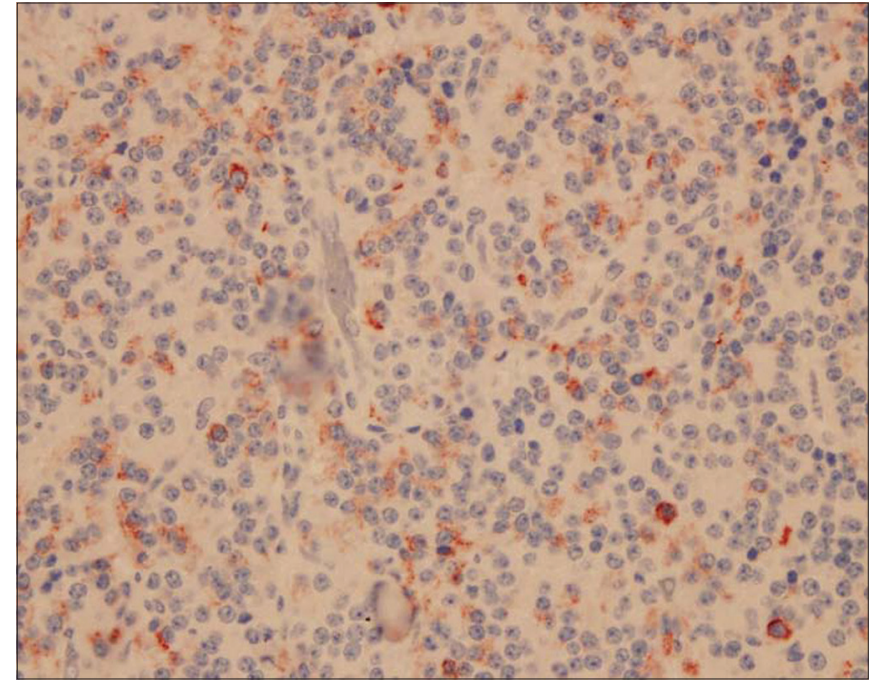

Fig. 4 Photomicrograph of tumour cells shows positive staining with insulin (Haematoxylin \& eosin, $\times$ 400).

T2-W and DW images, and as strong enhancement in dynamic contrast-enhanced studies.

More recently, functional imaging with radiolabelled isotopes such as octreotide is used as another tool for the detection and evaluation of insulinomas, with a sensitivity of $60 \%$ being reported by McAuley et al. ${ }^{(3)}$ EUS, an intraoperative imaging technique with a reported sensitivity of $93 \%{ }^{(3)}$ is however an invasive, operator-dependent procedure. Transhepatic portal venous sampling, another invasive procedure, has a reported sensitivity of $84 \%$ in detecting insulinomas. Compared to IACS, it is less sensitive ( $84 \%$ vs. $94 \%$ ), more invasive, and has possible serious complications such as intra-abdominal bleeding. ${ }^{(2,10)}$ However, it should be noted that the role of abdominal US, CT, MR imaging and EUS is not to diagnose insulinomas but to identify and localise tumour(s), and that nuclear medicine has a limited role in the evaluation of the operability of malignant lesions and the presence of metastatic disease. ${ }^{(3,10,14)}$

An insulinoma is often recognised as a small tumour that is difficult to locate via conventional imaging. Localisation of pancreatic insulinomas via IACS was first reported by Doppman et al in 1991. ${ }^{(6)}$ IACS plays a role in functional imaging and can also diagnose pancreatic insulinomas. ${ }^{(2,14,15)}$ A twofold or higher step-up from the baseline in the hepatic vein insulin concentration after arterial calcium injection constitutes a positive response, confirming the diagnosis of insulinoma. IACS is reported to be the most sensitive and powerful tool for preoperative tumour localisation in patients with insulinoma, and is helpful in the evaluation, management and treatment planning of insulinoma. ${ }^{(2,8)}$ In the case of our patient, malignant transformation of familial insulinoma could not be ruled out on the basis of CT and MR imaging because of the patient's uncommon clinical presentation (i.e. young age of 30 years and tumour size of approximately $6 \mathrm{~cm}$ ). In view of these factors, IACS was done to detect possible malignancy presenting as occult hepatic metastases or pancreatic lesions and to confirm the diagnosis of insulinoma before surgery was performed. Many surgeons emphasise the importance of preoperative localisation of insulinomas, as $10 \%-20 \%$ of these 
lesions are not detected during surgery and their presence may result in inappropriate surgical resection, thereby increasing the need for reoperation. Therefore, IACS plays an important role in preoperative localisation of insulinoma. ${ }^{(1)}$

Insulinoma can be treated by both medical and surgical management. Medical management is recommended for patients at high risk for surgery and for patients unwilling to undergo surgery. Other treatment options include dietary management and treatment with diazoxide, calcium channel blockers and somatostatin analogues. ${ }^{(1)}$ Surgical management has an extremely high success rate and is the treatment of choice for insulinomas. In this case, our patient showed symptom relief for three years after the tumour resection.

In conclusion, pancreatic insulinoma has specific CT and MR imaging findings. However, in the case of our patient who had an uncommon clinical presentation (i.e. a young age of 30 years and a large pancreatic tumour measuring $6 \mathrm{~cm}$ ), the possibility of malignant changes, such as the presence of hepatic metastases or occult pancreatic lesions, could not be ruled out. IACS is recommended for preoperative diagnosis of pancreatic insulinoma and treatment planning for patients with suspected malignant transformation.

\section{REFERENCES}

1. Abboud B, Boujaoude J. Occult sporadic insulinoma: Localization and surgical strategy. World J Gastroenterol 2008; 14:657-65.

2. Lo CY, Chan FL, Tam SC, et al. Value of intra-arterial calcium stimulated venous sampling for regionalization of pancreatic insulinomas. Surgery 2000; 128:903-9.
3. McAuley G, Delaney H, Colville J, et al. Multimodality preoperative imaging of pancreatic insulinomas. Clinical Radiology 2005; 60:1039-50.

4. Morganstein DL, Lewis DH, Jackson J, et al. The role of arterial stimulation and simultaneous venous sampling in addition to cross-sectional imaging for localization of biochemically proven insulinoma. Eur Radiol 2009; 19:2467-73.

5. Oberheim NA, Kim A, Farkas RL, et al. Giant pancreatic tumor with clinical characteristics of insulinoma but without common pathologic features. Endocr Pract 2011; 17:e12-6.

6. Doppman JL, Miller DL, Chang R, et al. Insulinomas: localization with selective intraarterial injection of calcium. Radiology 1991; 178:237-41.

7. Leow MK, Loh KC, Kon WY, et al. Clinical utility of selective intraarterial calcium-stimulated hepatic venous sampling in regionalisation of insulinomas - the Singapore experience. Ann Acad Med Singapore 2003; 32:86-91.

8. Tseng LM, Chen JY, Won JG, et al. The role of intra-arterial calcium stimulation test with hepatic venous sampling (IACS) in the management of occult insulinomas. Ann Surg Oncol 2007; 14:2121-7.

9. Guettier JM, Kam A, Chang R, et al. Localization of insulinomas to regions of the pancreas by intraarterial calcium stimulation: the NIH experience. J Clin Endocrinol Metab 2009; 94:1074-80.

10. Jackson JE. Angiography and arterial stimulation venous sampling in the localization of pancreatic neuroendocrine tumours. Best Pract Res Clin Endocrinol Metab 2005; 19:229-39.

11. Wiesli $P$, Brandle M, Schmid C, et al. Selective arterial calcium stimulation and hepatic venous sampling in the evaluation of hyperinsulinemic hypoglycemia: potential and limitations. J Vasc Interv Radiol 2004; 15:1251-6.

12. Oberg K. Pancreatic endocrine tumors. Semin Oncol 2010; 37:594-618.

13. Mittendorf EA, Liu YC, and McHenry CR. Giant insulinoma: case report and review of the literature. J Clin Endocrinol Metab 2005; 90:575-80.

14. Druce MR, Muthuppalaniappan VM, O'Leary B, et al. Diagnosis and localisation of insulinoma: the value of modern magnetic resonance imaging in conjunction with calcium stimulation catheterization. Eur J Endocrinol 2010; 162:971-8.

15. Chavan A, Kirchhoff TD, Brabant G, et al. Role of the intra-arterial calcium stimulation test in the preoperative localization of insulinomas. Eur Radiol 2000; 10:1582-6. 